\title{
Toll-like receptor 9-induced type I IFN protects mice from experimental colitis
}

\author{
Kyoko Katakura, ${ }^{1}$ Jongdae Lee, ${ }^{1}$ Daniel Rachmilewitz,, ${ }^{2}$ Gloria Li, ${ }^{3}$ Lars Eckmann, ${ }^{1}$ and Eyal Raz ${ }^{1}$ \\ 1Department of Medicine, UCSD, La Jolla, California, USA. ${ }^{2}$ Division of Medicine, Shaare Zedek Medical Center, Jerusalem, Israel. \\ ${ }^{3}$ Departments of Radiation Oncology and Medical Physics, Memorial Sloan Kettering Cancer Center, New York, New York, USA.
}

\begin{abstract}
Experimental colitis is mediated by inflammatory or dysregulated immune responses to microbial factors of the gastrointestinal tract. In this study we observed that administration of Toll-like receptor 9 (TLR9) agonists suppressed the severity of experimental colitis in $R A G 1^{-/-}$but not in SCID mice. This differential responsiveness between phenotypically similar but genetically distinct animals was related to a partial blockade in TLR9 signaling and defective production of type I IFN (i.e., IFN- $\alpha / \beta$ ) in SCID mice upon TLR9 stimulation. The addition of neutralization antibodies against type I IFN abolished the antiinflammatory effects induced by TLR9 agonists, whereas the administration of recombinant IFN- $\beta$ mimicked the antiinflammatory effects induced by TLR9 agonists in this model. Furthermore, mice deficient in the IFN- $\alpha / \beta$ receptor exhibited more severe colitis than wild-type mice did upon induction of experimental colitis. These results indicate that TLR9-triggered type I IFN has antiinflammatory functions in colitis. They also underscore the important protective role of type I IFN in intestinal homeostasis and suggest that strategies to modulate innate immunity may be of therapeutic value for the treatment of intestinal inflammatory conditions.
\end{abstract}

\section{Introduction}

The study of different animal models of intestinal inflammation has revealed that the interaction of susceptibility genes and microenvironmental factors such as intestinal microbiota leads to dysregulated mucosal immunity $(1,2)$. In particular, cytokine imbalance and the production of inflammatory mediators, mainly TNF- $\alpha$, have been implicated in the pathogenesis of experimental colitis and inflammatory bowel disease (IBD) in humans $(3,4)$.

Bacterial DNA and its synthetically derived immunostimulatory sequence oligodeoxynucleotide (ISS-ODN, also known as CpGODN) contain unmethylated $\mathrm{CPG}$ dinucleotide motifs within consensus sequences and are ligands of Toll-like receptor 9 (TLR9) (5). Like other TLR ligands such as LPS, ISS-ODN has a broad range of effects on the mammalian innate immune system. In particular, ISS-ODN induces the secretion of Th1-type cytokines and upregulates the expression of costimulatory molecules on antigenpresenting cells (6). These immunostimulatory characteristics of ISS-ODN have been utilized to elicit Th1-dependent immune responses (7) and mucosal immunity (8), leading to an enhanced host defense against invading pathogens (9).

TLR9 agonists (i.e., bacterial DNA or ISS-ODN) effectively prevent or ameliorate the severity of colonic inflammation in $\mathrm{CD}^{+}$ $\mathrm{T}$ cell-dependent (e.g., spontaneous colitis in $I L-10^{-/-}$mice) and $\mathrm{CD}^{+} \mathrm{T}$ cell-independent (e.g., dextran sulfate sodium-induced [DSS-induced]) models of experimental colitis $(10,11)$. The

Nonstandard abbreviations used: 129, 129/SvEv; B6, C57BL/6; B/c, BALB/c; $\mathrm{BMDM}$, bone marrow-derived macrophage; BM-MDC, bone marrow-derived myeloid dendritic cell; BM-PDC, bone marrow-derived plasmacytoid dendritic cell; DAI, disease activity index; DNA-PK, DNA-dependent protein kinase; DSS, dextran sulfate sodium; IBD, inflammatory bowel disease; IRF, interferon regulatory factor; ISS-ODN, immunostimulatory sequence oligodeoxynucleotide; mIFN, mouse IFN; MPO, myeloperoxidase; TLR, Toll-like receptor.

Conflict of interest: The authors have declared that no conflict of interest exists.

Citation for this article: J. Clin. Invest. 115:695-702 (2005)

doi:10.1172/JCI200522996 protective effects of ISS-ODN in the DSS-induced colitis model were transient, as readministration of DSS without ISS-ODN to mice previously treated with ISS and given DSS elicited colonic inflammation similar to that observed in mice injected with saline and given DSS (10). The transient nature of ISS-ODNmediated protection suggests that its administration does not elicit immunological memory. These protective effects are thus likely to be mediated by innate rather than adaptive immune responses (e.g., regulatory $\mathrm{T}$ cells).

To better understand the antiinflammatory role of TLR9-activated innate immunity in colonic inflammation, we initiated studies in T cell- and B cell-deficient animals, i.e., SCID and $R A G 1^{-/-}$mice. Surprisingly, our results indicated that while ISSODN administration inhibited colonic inflammation in $R A G 1^{-}$ mice, as was observed previously in wild-type animals $(10,11)$, it did not affect the severity of DSS-induced colitis in SCID mice. This differential response to the administration of TLR9 agonists between genetically distinct but phenotypically similar animals provided us with an opportunity to explore the cellular and the molecular events by which the activation of innate immunity can inhibit colonic inflammation. Our results indicate a major role for IFN- $\alpha / \beta$ in this process and reveal its protective function in intestinal homeostasis.

\section{Results}

TLR9 agonist attenuates DSS-induced colitis in RAG1-/- but not SCID mice. Previous studies demonstrated similar histopathological features in the colons of WT and SCID mice after the oral administration of DSS (12), a finding we confirmed in our work (Table 1). RAG1/-mice, which resemble SCID mice phenotypically, also showed a similar degree of colon inflammation after being fed DSS (Table 1). As expected, administration of ISS-ODN to WT mice attenuated DSS-induced colitis, as shown by improved disease activity and histological scores and a reduction in colonic myeloperoxidase (MPO) activity $(10,11)$. This protection was also observed in $R A G 1^{-/}$ mice on a C57BL/6 (B6) background but, interestingly, not in 


\section{Table 1}

Effect of administration of TLR9 agonists on DSS-induced colitis in WT, SCID, and $R A G 1^{-1-}$ mice

$\begin{array}{lcccc}\text { Mouse } & \text { Intervention } & \text { DAI } & \text { MPO (U/g) } & \text { HS } \\ \text { B6 (WT) } & \text { DSS } & 5.1 \pm 0.3 & 1.9 \pm 0.2 & 10.3 \pm 0.2 \\ \text { WT } & \text { DSS + ISS-1 } & 0.3 \pm 0.2^{\mathrm{A}} & 0.5 \pm 0.1^{\mathrm{A}} & 4.4 \pm 0.4^{\mathrm{A}} \\ \text { WT } & \text { DSS + M-ODN } & 6.6 \pm 1.1 & 2.0 \pm 0.3 & 10.3 \pm 0.5 \\ \text { SCID } & \text { DSS } & 5.7 \pm 0.5 & 1.7 \pm 0.4 & 9.6 \pm 0.7 \\ \text { SCID } & \text { DSS + ISS-1 } & 5.2 \pm 0.5 & 1.5 \pm 0.4 & 8.2 \pm 0.5 \\ \text { SCID } & \text { DSS + ISS-2 } & 6.5 \pm 0.6 & 1.3 \pm 0.1 & 9.8 \pm 0.3 \\ \text { RAG1-/- } & \text { DSS } & 7.1 \pm 0.2 & 2.1 \pm 0.1 & 8.6 \pm 0.6 \\ \text { RAG1-- } & \text { DSS + ISS-1 } & 0.8 \pm 0.2^{\mathrm{A}} & 0.5 \pm 0.1^{\mathrm{A}} & 3.9 \pm 0.4^{\mathrm{A}} \\ \text { RAG1-- } & \text { DSS + ISS-2 } & 1.9 \pm 0.7^{\mathrm{A}} & 0.9 \pm 0.2^{\mathrm{A}} & 5.0 \pm 0.8^{\mathrm{A}}\end{array}$

Mice (B6 background) were treated once s.c. with ISS-1 (1018), ISS-2 (D19), or control ODN (M-ODN) (1019), at a dose of $10 \mu \mathrm{g} / \mathrm{animal}, 2$ hours prior to the induction of colitis by DSS (1.5\%). Colitis was evaluated 7 days later. No mortality was observed in any of the experimental groups. Results are mean \pm SEM of at least 8 mice/group. ${ }^{A} P<0.05$ versus untreated group. HS, histological score.

SCID mice (B6) (Table 1). Histologically, the extensive ulcerations with mucosal inflammation induced by DSS were markedly attenuated in the colons of WT and $R A G 1^{-/-}$mice but not in the colons of SCID mice after the administration of ISS-ODN (Figure 1). Similar data were obtained in WT, $R A G 1^{-/}$, and SCID mice on a $\mathrm{BALB} / \mathrm{c}(\mathrm{B} / \mathrm{c})$ background (data not shown).

Soluble factors from ISS-ODN-stimulated $R A G 1^{-/-}$splenocytes inbibit DSS-induced colitis. Because the activation of TLR9 by its ligands has a broad range of effects on the mammalian innate immune system, the antiinflammatory effect induced by its activation in WT and $R A G 1^{-/-}$mice could be mediated by soluble factors secreted from effector cells or by cognate interactions between effector cells. To explore these possibilities, we generated conditioned medium from ISS-ODN-stimulated $R A G 1^{-/-}$splenocytes and evaluated its antiinflammatory effect on DSS-induced colitis in SCID mice, which were unresponsive to the effect of ISS-ODN. The administration of this conditioned medium attenuated the severity of DSS-induced colitis in SCID mice, whereas conditioned medium from ISS-ODN-stimulated SCID splenocytes, as well as control medium, had no effect on the outcome of colitis in these mice (Table 2). To determine whether the protective effects of the conditioned media depended on signaling through TLR, we used mice that lack the essential adaptor molecule MyD88 required for TLR9 signaling. These mice failed to respond to ISS-ODN administration when challenged with DSS, yet the administration of conditioned medium from ISS-ODN-stimulated $R A G 1^{-/-}$

\section{Figure 1}

Histological evaluation. (A-F) WT mice (A and B), RAG1-/- mice (C and $\mathbf{D}$ ), and SCID mice (E and F) were treated with ISS-ODN (DSS + ISS; A, C, and E) or were injected with saline (DSS; B, D, and F) and 2 hours later were given 1.5\% DSS in the drinking water. After 7 days, paraffin sections of the colon were prepared and stained with $\mathrm{H} \& \mathrm{E}$. In WT and $R A G 1^{-1-}$ mice, ISS treatment markedly attenuated colitis, as shown by the absence of mucosal ulceration and inflammatory cell infiltration in the same strain not treated with ISS (A and C). In contrast, SCID mice exhibited extensive mucosal ulceration and inflammatory cell infiltration into mucosa, submucosa, and muscle regardless of ISS treatment (E and $\mathbf{F})$. splenocytes resulted in attenuation of colitis in these mice (Table 2). Taken together, these data suggest the presence of soluble and inducible factors with antiinflammatory properties in conditioned medium from ISS-ODN-stimulated $R A G 1^{-/-}$splenocytes. These factors appear to bypass the partial or complete blockade of TLR9 signaling in SCID and MyD88-/- mice.

TLR9 triggers divergent type I IFN responses in SCID and RAG1-1mice. The data reported above indicate that the transfer of conditioned medium from ISS-ODN-stimulated $R A G 1^{-/-}$splenocytes, but not SCID splenocytes, to DSS-treated SCID mice is sufficient to inhibit colitis. To identify an inhibitor of colitis in the conditioned medium, we measured the levels of different soluble mediators known to be induced by ISS-ODN in splenocyte culture (6). As presented in Figure 2A, the levels of IL-12 (p40), IL-6, and IL-10 were similar in the supernatants of ISS-ODN-stimulated SCID and $R A G 1^{-/-}$splenocytes. In contrast, conditioned medium from ISS-ODN-treated SCID splenocytes had lower levels of IFN- $\gamma$ (2.5-fold), RANTES (2.3-fold), and IFN- $\alpha / \beta$ (6.3fold). To further confirm these differences in vivo, we injected ISS-ODN i.v. into the tail veins of SCID and $R A G 1^{-/-}$mice and assayed the levels of these cytokines in their serum (Figure 2B). Although SCID and $R A G 1^{-/-}$mice produced similar amounts of IL- 6 and IL-12, they differed in their levels of IFN- $\gamma$ (2-fold) and IFN- $\alpha / \beta$ (10-fold). The levels of IFN- $\alpha / \beta$ were analyzed by bioassay. We determined in preliminary studies that this assay was more sensitive by far than a commercially available ELISA (see Supplemental Figures 1 and 2; supplemental material available online with this article; doi:10.1172/JCI200522996DS1). Moreover, the reduced IFN- $\alpha / \beta$ production in SCID mice was not due to lower numbers of DCs $\left(\mathrm{CD} 11 \mathrm{c}^{+}\right)$or plasmacytoid DCs (PDCs) (CD11clo $\left./ \mathrm{B} 220^{+} / \mathrm{GR} 1^{+}\right)$, which are the major source of IFN- $\alpha / \beta$ in the mouse, as FACS analysis demonstrated that their numbers were similar in the spleens of SCID and $R A G 1^{-/-}$ mice (data not shown).

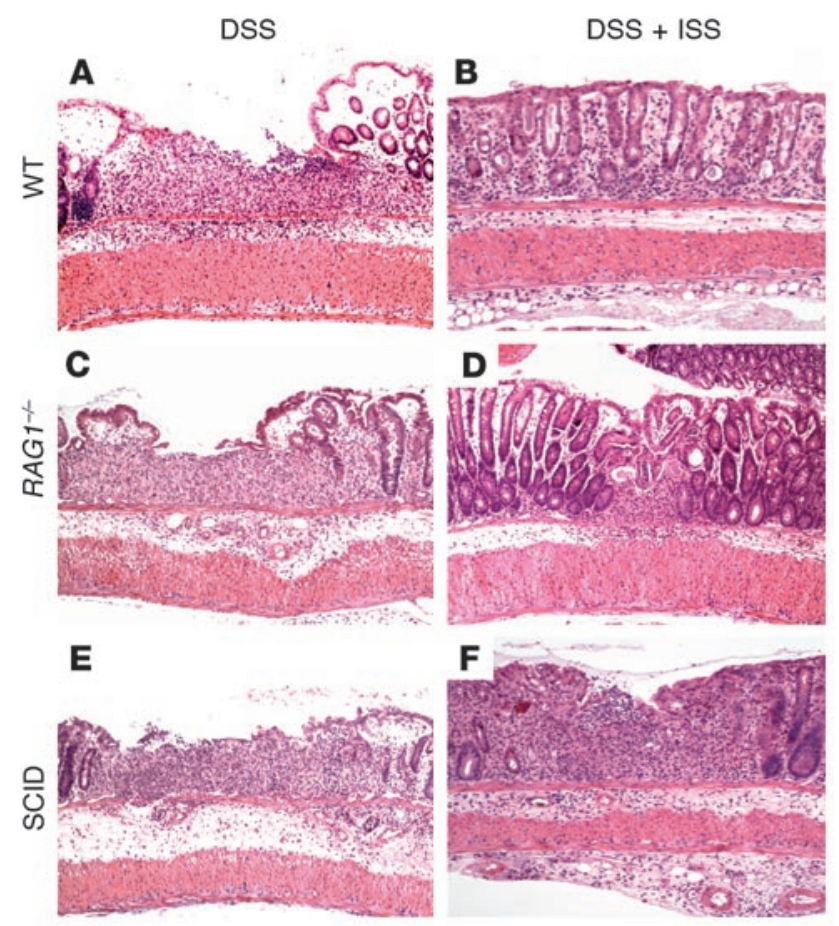


Table 2

Effect of ISS-ODN-conditioned medium on DSS-induced colitis in SCID and MyD $88^{-/-}$mice

\begin{tabular}{|c|c|c|c|c|}
\hline Mouse & Intervention & DAI & MPO (U/g) & HS \\
\hline SCID & $R A G 1^{-1-}$ control medium & $4.6 \pm 0.7$ & $2.0 \pm 0.3$ & $7.8 \pm 0.8$ \\
\hline SCID & RAG1-/-ISS-1-conditioned medium & $1.4 \pm 0.6^{A}$ & $0.2 \pm 0.1^{A}$ & $5.2 \pm 0.6^{A}$ \\
\hline SCID & SCID control medium & $5.8 \pm 0.5$ & $2.3 \pm 0.2$ & $8.1 \pm 0.6$ \\
\hline SCID & SCID ISS-1-conditioned medium & $6.8 \pm 0.4$ & $2.0 \pm 0.2$ & $9.6 \pm 0.4$ \\
\hline MyD88-/- & $R A G 1^{-1-}$ control medium & $4.4 \pm 0.5$ & $1.6 \pm 0.2$ & $7.6 \pm 1.1$ \\
\hline MyD $88^{-1-}$ & RAG1-1-ISS-1-conditioned medium & $0.5 \pm 0.3^{B}$ & $0.5 \pm 0.1^{\mathrm{B}}$ & $3.9 \pm 0.8^{B}$ \\
\hline MyD $88^{-1-}$ & Saline & $4.7 \pm 0.3$ & $1.8 \pm 0.4$ & $5.7 \pm 0.7$ \\
\hline MyD $88^{-/-}$ & ISS-1 & $4.5 \pm 1.0$ & $1.8 \pm 0.3$ & $6.8 \pm 1.4$ \\
\hline
\end{tabular}

Conditioned media were generated by incubation of $2.5 \times 10^{6}$ splenocytes $/ \mathrm{ml}$ from $R A G 1^{-/-}$or SCID mice with or without $10 \mu \mathrm{g} / \mathrm{ml}$ of ISS- 1 for 3 days. SCID or MyD88 ${ }^{-/-}$mice (B6) were treated i.p. with $200 \mu \mathrm{l} /$ mouse of control medium or ISS-1-conditioned medium 2 hours prior to the induction of colitis (1.5\% DSS) and on days 2, 4, and 6 after the induction of colitis. Colitis was evaluated on day 7 after induction. No mortality was observed in any of the experimental groups ( $n=6$ mice per group). Data are mean \pm SEM and represent 1 of 3 experiments with similar results. ${ }^{A} P<0.05$ versus $R A G 1^{-/}$control medium-treated group. ${ }^{\mathrm{B} P}<0.05$ versus $M y D 88^{-/}$control medium-treated group.

of ISS-ODN to IFN- $\alpha / \beta R^{-/-}$mice that had received $4 \%$ DSS increased their mortality from $50 \%$ to $75 \%$ $(P=\mathrm{NS})$, whereas the administration of ISS-ODN to WT animals that had received $4 \%$ DSS inhibited the severity of the various parameters of colonic inflammation (Table 4). To test whether IFN- $\alpha / \beta$ directly prevents the damage inflicted by DSS in the colon, we injected recombinant mouse IFN- $\beta$ $(\operatorname{mIFN}-\beta)$ i.p. on days $0,2,4$, and 6 after DSS administration. As shown in Table 5, injection of recombinant mIFN- $\beta$ decreased the disease activity and the histological scores of DSS-treated mice. Taken together, these data indicate that the antiinflammatory effects of ISS-ODN in this system were mediated largely by type I IFN.

Finally, to explore whether the protective role of IFN- $\alpha / \beta$ is mediated via the inhibition of inflammatory cells, such as macrophages, we adoptively transferred bone marrow-derived macrophages (BMDMs) from WT or IFN- $\alpha / \beta R^{-/-}$ mice i.p. into WT mice prior to challenge of the recipient mice with DSS. Administration of ISS-

IFN- $\alpha / \beta$ protects from DSS-induced colitis. As IFN- $\alpha / \beta$ was induced by ISS-ODN in $R A G 1^{-/-}$but not in SCID mice, we hypothesized that IFN- $\alpha / \beta$ might play a role in suppressing the colitis elicited by DSS administration. As presented in Table 3, injection of neutralizing anti-IFN- $\alpha / \beta$ was sufficient to abolish the beneficial effect induced by ISS-ODN on colitis, whereas the injection of control IgG Abs had no effect.

To further evaluate the protective role of ISS-induced IFN- $\alpha / \beta$ against DSS-induced colitis, we treated mice lacking the common receptor for IFN- $\alpha / \beta$, i.e., IFN- $\alpha / \beta R^{-/-}$mice, with ISS-ODN. IFN- $\alpha$ / $\beta R^{-/-}$mice were extremely sensitive to DSS-induced colitis. The administration of $4 \%$ DSS to $I F N-\alpha / \beta R^{-/-}$mice resulted in $50 \%$ mortality, whereas no mortality was observed for the WT control mice $(P<0.05)$. The administration of $2.5 \%$ DSS colitis to IFN- $\alpha$ ) $\beta R^{-/-}$mice resulted in the same severity of colitis as that induced by $4 \%$ DSS in WT mice, whereas the administration of $2.5 \%$ DSS to WT mice did not induce apparent colitis $(P>0.05$ for the disease activity index [DAI] and MPO). Interestingly, the administration
ODN protected WT mice that received adoptively transferred WT BMDMs from experimental colitis but did not protect WT mice that received adoptively transferred $I F N-\alpha / \beta R^{-/-}$BMDMs (Table 6). In another set of experiments, BMDMs from WT and from $I F N-\alpha / \beta R^{-/-}$mice were labeled with CFSE prior to their transfer into WT mice. The number of CFSE-labeled BMDMs in the colons of DSS-challenged, ISS-ODN-treated WT mice was comparable in mice adoptively transferred with either WT or $I F N-\alpha / \beta R^{-/-}$mice, suggesting that BMDMs from WT and IFN- $\alpha$ / $\beta R^{-/-}$mice trafficked equally to the inflamed colon (data not shown). These results indicate that IFN- $\alpha / \beta$ inhibits, at least in part, the severity of DSS-induced colitis by suppressing macrophage proinflammatory activity.

Regulation of IFN- $\alpha / \beta$ production via TLR9 depends on DNA-dependent protein kinase. As outlined above, IFN- $\alpha / \beta$ is differentially induced in SCID and $R A G 1^{-/-}$mice upon TLR9 signaling. Previous studies have demonstrated that NF-KB, MAPK, and members of the interferon regulatory factor (IRF) family control IFN- $\alpha / \beta$ transcription

\section{Figure 2}

Cytokine production after ISS-ODN stimulation. (A) Splenocytes $\left(10^{6} / \mathrm{ml}\right)$ from $R A G 1^{-/-}$ and SCID mice were stimulated with ISS-1 (10 $\mu \mathrm{g} / \mathrm{ml}$ ) and cytokine levels in the supernatants were measured 48 hours later. (B) Serum levels of cytokines 2 hours after i.v. injection of $50 \mu \mathrm{g}$ of ISS-1. Results represent 1 of 2 experiments ( $n=4$ per group per experiment). Similar results were obtained for ISS-2 (data not shown). The cytokine levels in naive mice were below detectable levels (data not shown). ${ }^{*} P<0.05$ versus $R A G 1^{-1-}$.
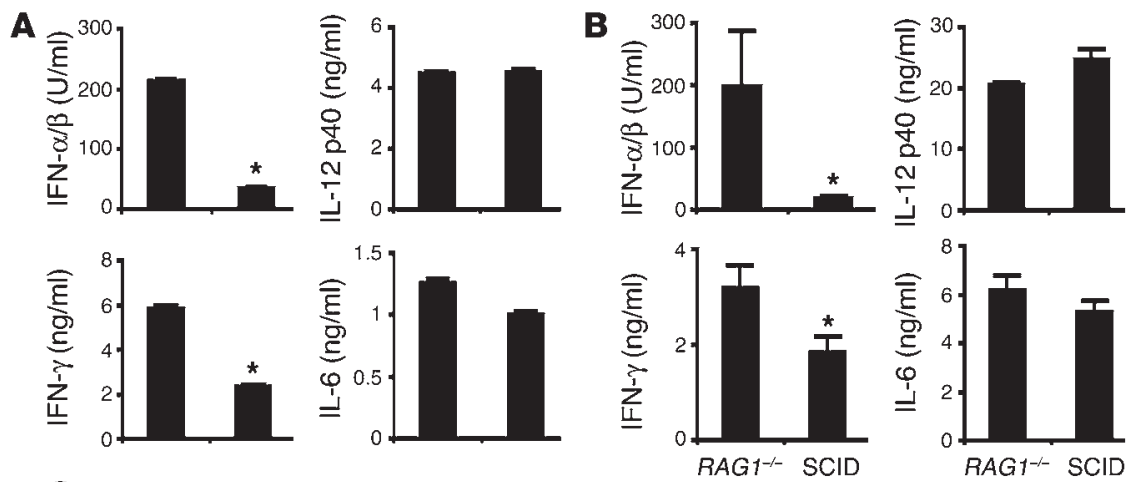

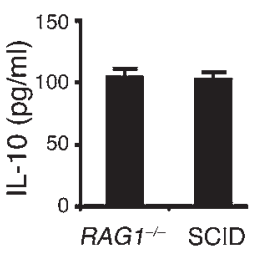




\section{Table 3}

TLR9-induced type I IFN protects mice from experimental colitis

\begin{tabular}{lllll} 
Mouse & \multicolumn{1}{c}{ Intervention } & \multicolumn{1}{c}{ DAI } & MPO (U/g) & \multicolumn{1}{c}{ HS } \\
RAG1- & None & $7.4 \pm 0.2$ & $1.9 \pm 0.4$ & $9.1 \pm 0.3$ \\
RAG1-- & ISS-1 & $0.8 \pm 0.2^{\mathrm{A}}$ & $0.5 \pm 0.1^{\mathrm{A}}$ & $4.6 \pm 0.5^{\mathrm{A}}$ \\
RAG1-- & ISS- $1+$ anti-IFN- $\alpha / \beta$ & $6.8 \pm 0.7$ & $1.7 \pm 0.2$ & $9.0 \pm 0.3$ \\
RAG1-- & ISS-1 + control IgG & $0.8 \pm 0.4 \mathrm{~B}$ & $0.7 \pm 0.2^{\mathrm{B}}$ & $3.7 \pm 0.6^{\mathrm{B}}$
\end{tabular}

RAG1/- mice (B6) were injected i.v. with $25 \mu \mathrm{g} /$ mouse of rabbit anti$\mathrm{mIFN}-\alpha / \beta(8,000$ IFN- $\alpha / \beta-$ neutralizing units) or with control rabbit IgG $(25 \mu \mathrm{g} / \mathrm{mouse})$ before treatment with ISS-1 (10 $\mu \mathrm{g} / \mathrm{animal})$. Colitis was induced by $1.5 \%$ DSS in drinking water and was evaluated 7 days later. No mortality was observed in any of the experimental groups ( $n=6$ mice per group). Data are mean \pm SEM and represent 1 of 2 experiments with similar results. ${ }^{A} P<0.05$ versus untreated group. ${ }^{\mathrm{B}} P<0.05$ versus anti-IFN- $\alpha / \beta$-treated group.

(13). Since deletion of the kinase domain of the catalytic subunit of DNA-dependent protein kinase (DNA-PK) is responsible for the SCID phenotype, we investigated the role of DNA-PK in TLR9mediated type I IFN induction. To do so, we incubated bone marrow-derived myeloid dendritic cells (BM-MDCs) from $R A G 1^{-/-}$ mice with various concentrations of NU7026, a DNA-PK inhibitor (Calbiochem), with or without ISS-ODN. This compound selectively inhibited IFN- $\alpha / \beta$ without affecting IL-12 (p40) production by ISS-ODN (Supplemental Figure 3), suggesting selective involvement of DNA-PK in the induction of type I IFN by TLR9 triggering. Similarly, BM-MDCs from $R A G 1^{-/-}$but not from SCID mice produced high levels of IFN- $\alpha / \beta$ upon ISS-ODN stimulation (Figure 3A). Comparable results were obtained for bone marrow-derived plasmacytoid dendritic cells (BM-PDCs; data not shown). This divergent induction of IFN- $\alpha / \beta$ was paralleled by nuclear translocation of IRF-1 and IRF-8 in $R A G 1^{-/-}$but not in SCID mice (Figure $3 \mathrm{~B}$ ). In contrast, the activation of NF- $\mathrm{KB}$, MAPK, IRF-3, and IRF-7 was similar in BM-MDCs obtained from either $R A G 1^{--}$or SCID mice (Figure 3B), indicating a specific signaling blockade downstream of TLR9 in SCID but not in RAG1/BM-MDCs. Furthermore, as assessed by the phosphorylation of GST-p53, DNA-PK was rapidly activated by ISS-ODN in BMMDCs from $\mathrm{RAG1}^{-{ }^{-}}$but not from SCID mice (Figure 3B). In contrast to the activation by the TLR9 agonist, the stimulation of BM-MDCs with the TLR 4 agonist LPS resulted in IFN- $\alpha / \beta$ production (Figure 3C) and activation of NF-KB, MAPK, and IRF molecules (Figure 3D) that was similar in BM-MDCs from SCID and $R A G 1^{-/-}$mice. We also observed defects in activation of IRF-1 and IRF-8 in BM-PDCs from DNA-PK $K^{-1-}$ mice (Figure $4 \mathrm{~A}$ ) as well as very low levels of IFN- $\alpha / \beta$ after ISS-ODN stimulation in BMPDCs and BM-MDCs from DNA-PK- $\mathrm{K}^{--}$mice (Figure 4, B and C, and Supplemental Tables 1 and 2). Thus, the data obtained with the DNA-PK inhibitor with the SCID and the DNA-PK ${ }^{-/-} \mathrm{BM}$ MDCs and BM-PDCs indicate the necessary involvement of DNAPK in the induction of IFN- $\alpha / \beta$ via TLR9 signaling.

$D N A-P K$ is positioned downstream of MyD88. As TLR9 signaling depends on MyD88 (5) and TLR9-mediated IFN- $\alpha / \beta$ production depends on DNA-PK (Figures 3 and 4), we evaluated the potential relationship between MyD88 and DNA-PK in the TLR9 signaling pathway. DNA-PK was selectively activated by ISS-ODN but not by LPS in WT-derived BM-MDCs, but ISS-ODN did not activate DNA-PK in MyD88-/-derived BM-MDCs (Figure 4D). Recently, it was suggested that the induction of IFN- $\alpha / \beta$ via TLR9 requires the formation of a complex consisting of MyD88, TRAF, and IRF-7, as well as TRAF6-dependent ubiquitination of IRF-7 $(14,15)$. As shown in Figure 4D, we could detect ubiquitination of IRF-7 in $R A G 1^{-/-}$but not SCID BM-MDCs. Taken together, these data further indicate the involvement of DNA-PK in the translocation of IRF-1/IRF-8 and in the ubiquitination of IRF-7 upon TLR9 signaling and indicate that DNA-PK activation occurs downstream of MyD88 in this signaling pathway (Figure 4E).

\section{Discussion}

TLRs function as sentinels of innate immunity (16). By recognizing signature microbial compounds they trigger the transcriptional activation of proinflammatory cytokines (e.g., IL-12 and TNF- $\alpha$ ) and chemokines (e.g., RANTES), as well as costimulatory molecules (e.g., CD40) (17). The activation of this proinflammatory program initiates defense mechanisms that are vital for host survival $(16,17)$. Indeed, various $T L R^{-/-}$mice as well as mice deficient in TLR-related adaptor proteins (e.g., MyD88) fail to mount protective responses and succumb to various microbial infections (18).

In contrast to the currently accepted paradigm regarding the proinflammatory role of TLR-activated innate immunity (16, 17), our previous data indicated that TLR9 signaling results in the activation of an antiinflammatory program that attenuates inflammation in different models of experimental colitis (10, 11). In our studies presented here, we found that 2 genetically distinct but phenotypically similar strains of mice responded differently to ISS-ODN administration. While DSS-induced colitis in $R A G 1^{-/-}$mice was responsive to ISS-ODN, colitis in SCID mice was not. Here we utilized those ISS-responsive and ISSresistant phenotypes to better understand the antiinflammatory role of TLR9 signaling in colonic inflammation. Analysis of the response to ISS-ODN of these 2 mouse strains revealed defective TLR9-induced IFN- $\alpha / \beta$ production in SCID mice, which together with the IFN- $\alpha / \beta$ neutralization data indicated that type I IFN plays a major role in the inhibition of DSS-induced colonic inflammation. In addition, the lack of inhibition of DSS-induced colitis in ISS-ODN-treated WT mice that received adoptively transferred BMDMs from $I F N-\alpha / \beta R^{-/-}$but not in those that received BMDMs from WT mice (Table 6) also suggests that TLR9-induced type I IFN inhibits the inflammatory response of activated macrophages. Finally, we observed that

\section{Table 4}

IFN- $\alpha / \beta R^{-1-}$ mice are hypersensitive to DSS administration

\begin{tabular}{|c|c|c|c|c|}
\hline Mouse & Intervention & DAI & MPO (U/g) & HS \\
\hline WT & DSS & $5.4 \pm 0.5$ & $2.0 \pm 0.2$ & $8.8 \pm 1.1$ \\
\hline WT & DSS + ISS-1 & $0.1 \pm 0.1^{\mathrm{A}}$ & $0.2 \pm 0.01^{A}$ & $1.7 \pm 0.4^{\mathrm{A}}$ \\
\hline IFN- $\alpha / \beta R^{-/-}$ & DSS & $7.2 \pm 0.3$ & $2.0 \pm 0.1$ & $10.4 \pm 0.4$ \\
\hline IFN- $\alpha / \beta R^{-/-}$ & DSS + ISS- 1 & $7.8 \pm 0.2$ & $1.7 \pm 0.1$ & $10.7 \pm 0.4$ \\
\hline
\end{tabular}

WT and mutant mice were treated once s.c. with ISS-1 (10 $\mu \mathrm{g} / \mathrm{animal})$ 2 hours prior to the induction of colitis by $4 \%$ DSS. Colitis was evaluated 7 days later. $n=8$ animals per group for WT mice and $n=16$ per group for mutant mice. No mortality was observed in ISS-1-treated or -untreated WT mice. In contrast, ISS-1-treated mutant mice displayed mortality of $75 \%$, whereas ISS-1-untreated mutant mice displayed mortality of $50 \%$. The analysis was performed on surviving mice. Data are mean \pm SEM and represent 1 of 2 experiments with similar results. A $P<0.05$ versus untreated group. 
Table 5

Type I IFN protects from DSS-induced colitis

\begin{tabular}{lcccc} 
Mouse & Intervention & DAI & MPO (U/g) & HS \\
SCID & DSS & $7.7 \pm 0.3$ & $1.5 \pm 0.5$ & $11.1 \pm 0.2$ \\
SCID & DSS + IFN- $\beta$ & $4.6 \pm 0.9^{A}$ & $1.2 \pm 0.1$ & $6.8 \pm 1.2^{A}$ \\
\hline
\end{tabular}

SCID mice (B6) were treated i.p. with $1,000 \mathrm{U}$ of recombinant mIFN- $\beta$ 2 hours prior to and on days 2, 4, and 6 after the induction of colitis with $1.5 \%$ DSS. Colitis was evaluated 7 days after induction. No mortality was observed in any of the experimental groups. $n=8$ mice per group. Data are mean \pm SEM and represent 1 of 2 experiments with similar results. ${ }^{A} P<0.05$ versus untreated group.

IFN- $\alpha / \beta R^{-/-}$mice were extremely sensitive to colitis inflicted by DSS (Table 4) and that the administration of ISS-ODN to these mice increased their mortality. These data indicate that type I IFN has a physiological and protective role in colonic injury and that it also cross-regulates the other proinflammatory activities induced by TLR9 triggering. Indeed, in preliminary studies we observed that basolateral administration of IFN- $\alpha$ to polarized monolayers of intestinal epithelial cells protected the cells against apoptosis and disruption of the epithelial tight junctions (data not shown). Thus, type I IFN may protect against colonic inflammation by preventing epithelial barrier dysfunction.

As our results indicated that the SCID mutation confers resistance to the antiinflammatory effects of ISS-ODN on experimental colitis, we explored the signaling pathway that leads to the impaired production of IFN- $\alpha / \beta$. As presented in Figure 3, TLR9 stimulation of BM-MDCs from $R A G 1^{-1-}$ but not from SCID mice resulted in the activation of IRF-1 and IRF-8. Furthermore, we could detect ubiquitination of IRF-7, which is required for IFN- $\alpha$ production, in $R A G 1^{-/-}$but not in SCID BMPDCs (Figure 4). This signaling defect was specific for TLR9 activation, as TLR4 triggering resulted in a similar signaling profile and comparable IFN- $\alpha / \beta$ production in both $R A G 1^{-/-}$and SCID BM-PDCs (Figures 3 and 4). Our findings are supported by 2 recent studies that indicated an essential role for IRF-8 in the production of IFN- $\alpha / \beta$ after TLR9 stimulation. In those studies, the genetic deletion of IRF- 8 resulted in impaired IFN- $\alpha / \beta$ production by TLR9-triggered BM-MDCs and BM-PDCs (19, 20). Our results add to other previous observations indicating a role for DNA-PK in the activation of innate immunity (21-23) and demonstrate the involvement of DNA-PK after TLR9 triggering in the translocation of IRF-1/IRF- 8 and in the ubiquitination of IRF-7 (15). Our results also position DNA-PK downstream of the adaptor protein MyD88 in the TLR9 signaling pathway (Figures 4). However, in contrast to the data presented here, previous studies did not identify differences in IFN- $\alpha$ production after TLR9 stimulation in DNA-PK $K^{-/}$and WT mice $(24,25)$. Although we have no definitive explanation for these differences, they may be related to qualitative and quantitative differences in the specificity and sensitivity of detection of type I IFN, which consists of large family of related proteins, by bioassay used in our study versus the ELISA kit $(\operatorname{IFN}-\alpha)(24,25)$.

Type I IFN (IFN- $\alpha / \beta)$ was first described as an antiviral protein that exerts many regulatory effects on the immune response (26). Recent data support the concept of crossregulation of IFN- $\alpha / \beta$ and TNF- $\alpha$ in various inflammatory settings (27). This cross-regulation has been implicated in the aggravation (by TNF- $\alpha$ ) and the suppression (by IFN- $\beta$ ) of experimental allergic encephalitis (EAE), an animal model of multiple sclerosis. IFN- $\beta^{-/-}$mice are more susceptible to EAE (28), and the administration of IFN- $\beta$ to mice with EAE inhibits disease progression (29). On the other hand, TNF- $\alpha$ inhibits whereas TNF- $\alpha$ antagonists enhance IFN- $\alpha / \beta$ production by PDCs after viral exposure (27). Thus, it is conceivable that TNF- $\alpha$ / IFN- $\alpha / \beta$ cross-regulation may also play a major role in the regulation of intestinal inflammation. Based on the data presented in our study here, we propose that the beneficial effects of TNF- $\alpha$ antagonists in patients with $\operatorname{IBD}(3,30)$ are due in part to an increase in responsiveness to IFN- $\alpha / \beta$ in these patients. Interestingly, initial clinical studies have recently confirmed the beneficial effects of IFN- $\alpha / \beta$ in IBD; however, its therapeutic effect in this setting requires further validation (31).

A recent study documented the protective effect of TLR ligands in colonic injury (32). Our previous studies identified the antiinflammatory effects of TLR9 agonists in experimental colitis $(10,11)$. The results of our study here demonstrated that TLRinduced type I IFN mediates these protective effects on colonic inflammation. These findings and the hypersensitivity to DSS observed in $I F N-\alpha / \beta R^{-/-}$mice expand the already known range of activities of type I IFN and indicate an important role for type I IFN in intestinal homeostasis. Finally, our results suggest that strategies designed to trigger an IFN- $\alpha / \beta$ response may be of therapeutic value in intestinal inflammatory conditions.

\section{Methods}

\section{Reagents}

The following materials were obtained from commercial sources: DSS (molecular weight, 30-50 kDa), from ICN; Hemoccult, from Beckman Coulter; rabbit anti-mIFN- $\alpha$ and rabbit anti-mIFN- $\beta$, from PBL Biomedical Laboratories; rabbit IgG, from Jackson ImmunoResearch Laboratories; recombinant mIFN- $\beta$, from Chemicon International; LPS (Salmonella minnesota R595), from Alexis.

\section{ODNs}

The ODNs used in this study were LPS-free, HPLC-purified, single-stranded ODNs. Phosphorothioate-stabilized ISS-1 (class B, 1018) (5'-TGACTGTGAACGTTCGAGATGA-3'), M-ODN (1019) (5'-TGACTGTGAAGGTTC-

\section{Table 6}

ISS-ODN affects the severity of DSS-induced colitis by BMDMs

\begin{tabular}{lccccc} 
Mouse & BMDMs & Intervention & \multicolumn{1}{c}{ DAI } & MPO (U/g) & \multicolumn{1}{c}{ HS } \\
WT & WT & DSS & $5.2 \pm 0.6$ & $2.4 \pm 0.5$ & $9.8 \pm 0.2$ \\
WT & WT & DSS + ISS-1 & $0.7 \pm 0.4^{\mathrm{A}}$ & $0.8 \pm 0.2^{\mathrm{A}}$ & $3.0 \pm 0.4^{\mathrm{A}}$ \\
WT & $I F N-\alpha / \beta R^{-/}$ & DSS & $4.5 \pm 0.8$ & $2.2 \pm 0.4$ & $9.6 \pm 0.3$ \\
WT & $I F N-\alpha / \beta R^{-/-}$ & DSS+ISS- 1 & $3.3 \pm 1.3$ & $1.9 \pm 0.5$ & $8.5 \pm 0.5$ \\
\hline
\end{tabular}

ISS-ODN administration does not affect the severity of DSS-induced colitis in WT mice adoptively transferred with BMDMs from IFN- $\alpha / \beta R^{-/-}$mice. BMDMs $\left(5 \times 10^{6} /\right.$ mouse) from WT or from IFN- $\alpha / \beta R^{-/-}$mice were adoptively transferred i.p. into WT mice (129) and recipient mice were treated once s.c. with ISS-1 (10 $\mu \mathrm{g} / \mathrm{animal}) 2$ hours prior to the induction of colitis by DSS (4\%). Colitis was evaluated on day 7 after DSS administration. $n=8$ mice per group. No mortality was observed in any of the experimental groups. Data are mean \pm SEM and represent 1 of 2 experiments with similar results. ${ }^{A} P<0.05$ versus untreated group. 

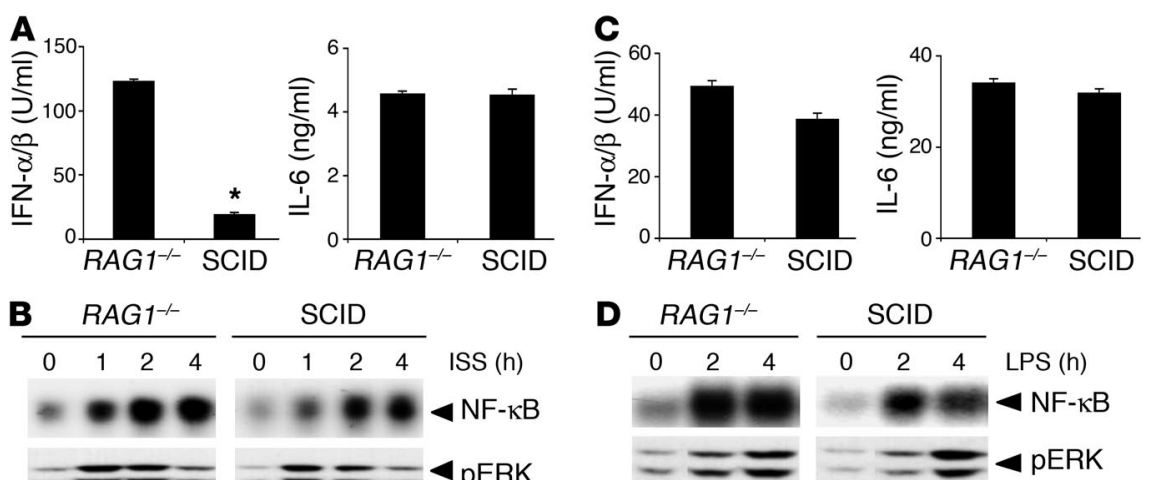

SCID
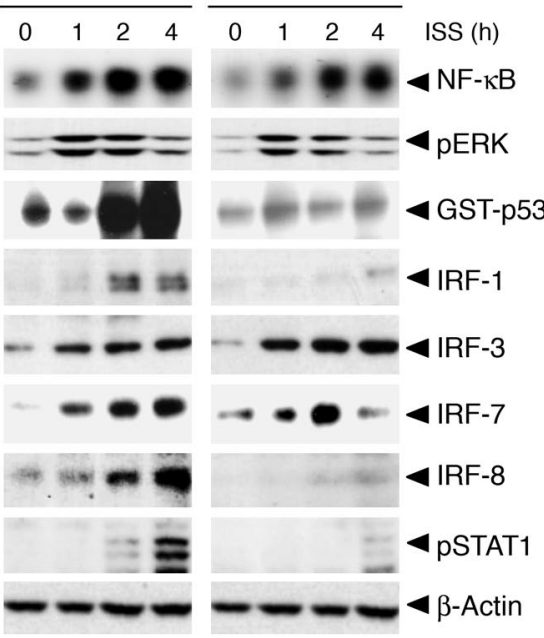

D $R A G 1^{-1}$

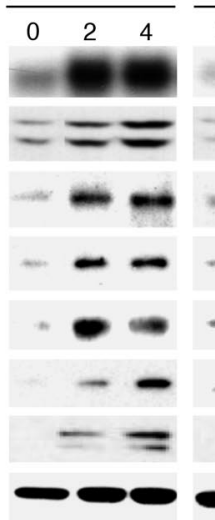

SCID

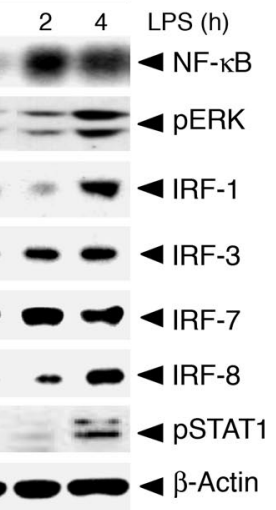

\section{Figure 3}

DNA-PK mediates TLR9-induced type I IFN production via IRF-1 and IRF-8. (A) BMMDCs (10\%/ml) from $R A G 1^{-/-}$and SCID mice (B6) were stimulated with ISS-1 $(10 \mu \mathrm{g} / \mathrm{ml})$. Cytokine levels in the supernatants were measured 24 hours later. ${ }^{*} P<0.05$ versus $R A G 1^{-1-}$. (B) BM-MDCs were treated with ISS-1 (10 $\mu \mathrm{g} / \mathrm{ml}$ ) and nuclear extracts were prepared. NF- $\mathrm{KB}$ was detected by electrophoretic mobility-shift assay (EMSA); activation of DNA-PK, by in vitro kinase assay; and activation of IRFs, pSTAT1, and pERK, by Western blotting. Results represent 1 of 3 experiments. (C) BM-MDCs $\left(10^{6} / \mathrm{ml}\right)$ from $R A G 1^{-/-}$and SCID mice (B6) were stimulated with $50 \mathrm{ng} / \mathrm{ml}$ of LPS. After 24 hours, the cytokine levels in the supernatants were measured. (D) BM-MDCs were treated with LPS $(50 \mathrm{ng} / \mathrm{ml})$ and nuclear extracts were prepared. Signaling assays were performed as described for $\mathbf{B}$. Results represent 1 of 3 experiments.
CAGATGA-3') (7), and the phosphodiester- and phosphorothioate-modified ISS-2 (class A, D19) (5'-GgtgcatcgatgcaGGGGGg-3') $(24,25)$ were purchased from TriLink BioTechnologies. Underlining indicates the differences between the sequences. Uppercase and lowercase letters indicate a base with a phosphorothioate- or a phosphodiester-modified backbone, respectively.

\section{Mice}

The SCID mice are homozygous for a mutation (scid) in DNA-PK (33). Specific pathogen-free B6, SCID (B6), and $R A G 1^{-/-}(\mathrm{B} 6)$ mice and $\mathrm{B} / \mathrm{c}$, $\operatorname{SCID}(\mathrm{B} / \mathrm{c})$, and $R A G 1^{-/-}(\mathrm{B} / \mathrm{c})$ mice $6-8$ weeks of age were purchased from Jackson Laboratory. 129/SvEv (129) and A.129 (IFN- $\left.\alpha / \beta R^{-/-}\right)$mice were purchased from B\&K Universal Ltd. $M y D 88^{-/-}$mice were kindly provided by S. Akira (Osaka University, Osaka, Japan), and are currently bred in our vivarium. Six- to eight-week-old $D N A-P K^{-/-}$mice (B6) (34) were generated and bred at Memorial Sloan Kettering Cancer Center. All experimental procedures were approved by the institutional committee for animal care and use of UCSD.

\section{Cell cultures and adoptive transfer}

BMDMs from WT and IFN- $\alpha / \beta R^{-/-}$mice were prepared and allowed to differentiate in macrophage medium supplemented with $30 \% \mathrm{~L}$-cell medium for 7 days as described (35). BM-MDCs were cultured from the long bones of WT, $R A G 1^{-/-} \mathrm{SCID}$, and DNA-PK $\mathrm{K}^{-/-}$mice in the presence of murine GMCSF (BD Biosciences - Pharmingen) and were characterized as described (36). BM-PDCs were cultured similarly in the presence of $100 \mathrm{ng} / \mathrm{ml}$ of human Flt3 ligand (PeproTech Inc.), as described $(24,25)$.

For adoptive transfer-based experiments, BMDMs $\left(5 \times 10^{6}\right.$ cells per mouse) were transferred i.p. into WT mice just prior to ISS-ODN injection and 2 hours before DSS administration.

\section{Induction and evaluation of experimental colitis}

In a previous study we identified the concentration of DSS required to induce a similar severity of colitis in the different mouse strains used in this study (11). Mice on the B6 background were given $1.5 \%$ (weight/volume) DSS, mice on the 129 background were given $4 \%$ DSS, and mice on the $\mathrm{B} / \mathrm{c}$ background were given $3.5 \%$ DSS; for all, the DSS was dissolved in sterile, distilled water and was provided ad libitum for 7 days (11). Groups of mice were treated with $10 \mu \mathrm{g} /$ mouse of ODN s.c. 2 hours before DSS administration. The DAI (the combined score of weight loss and bleeding) was determined as described $(10,11)$. Briefly, scores are defined as follows: for loss in body weight, $0=$ no loss, $1=5-10 \%$, $2=10-15 \%, 3=15-20 \%, 4=$ over $20 \%$; for hemoccult, $0=$ no blood, $2=$ positive, and $4=$ gross blood.

\section{Determination of MPO activity}

Colon tissues were opened longitudinally and a 50-mg portion was homogenized in hexadecyltrimethyl-ammonium bromide $(0.5 \%)$ in $50 \mathrm{mmol} / \mathrm{l}$ phosphate buffer, $\mathrm{pH}$ 6.0. The homogenate was sonicated for 10 seconds, frozen and thawed 3 times, and centrifuged for 15 minutes. An aliquot of the supernatant was used for determination of enzyme activity as described $(10,11)$.

\section{Histological scoring}

After 7 days of DSS administration, mice were killed and the entire colon was excised, opened longitudinally, rolled onto a wooden stick, fixed with Bouin's Solution (Sigma-Aldrich), and embedded in paraffin. Tissue sections $(5 \mu \mathrm{m})$ were prepared, deparaffinized, and stained with hematoxylin and eosin. Histological scores were assigned by experimenters "blinded" to sample identity. Colonic epithelial damage was assigned scores as follows: 0 = normal; 1 = hyperproliferation, irregular crypts, and goblet cell loss; 2 = mild to moderate crypt loss (10-50\%); 3 = severe crypt loss (50-90\%); 4 = complete crypt loss, surface epithelium intact; 5 = small- to medium-sized ulcer ( $<10$ crypt widths); $6=$ large ulcer ( $\geq 10$ crypt widths). Infiltration with inflammatory cells was assigned scores separately for mucosa $(0=$ normal, $1=$ mild, $2=$ modest, $3=$ severe $)$, submucosa $(0=$ normal, $1=$ mild to modest, $2=$ severe $)$, and muscle $/$ serosa $(0=$ normal, $1=$ moderate to severe $)$. Scores for epithelial 


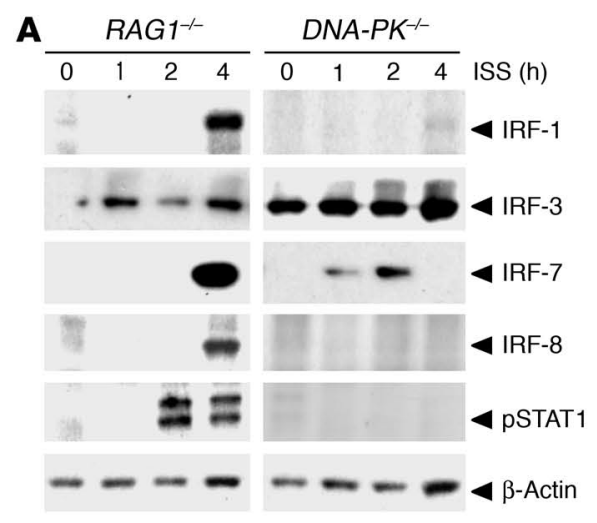

C

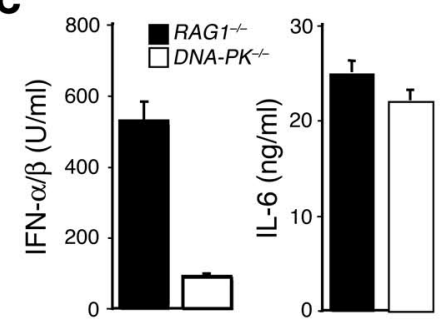

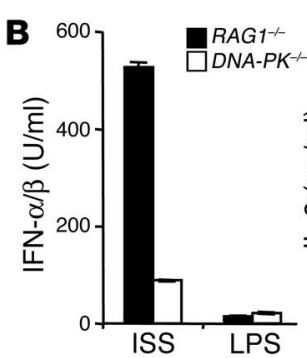
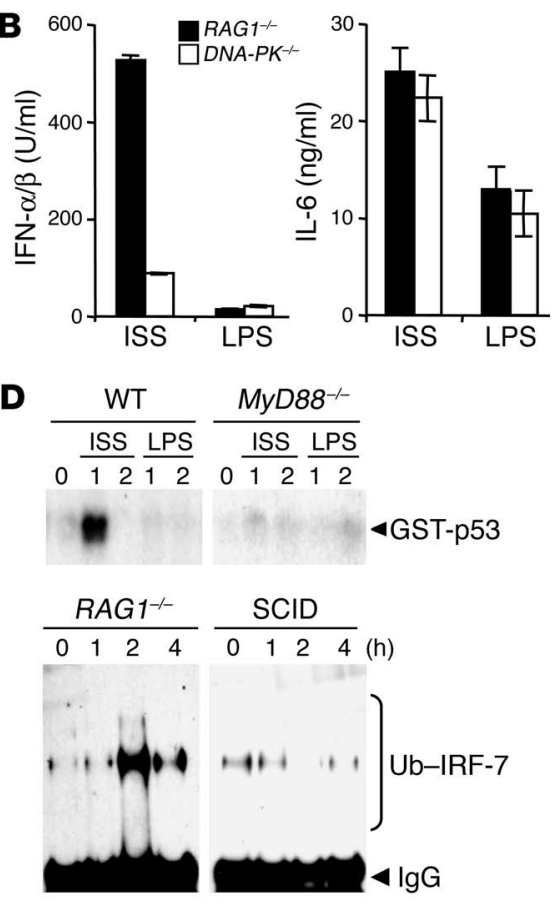

E

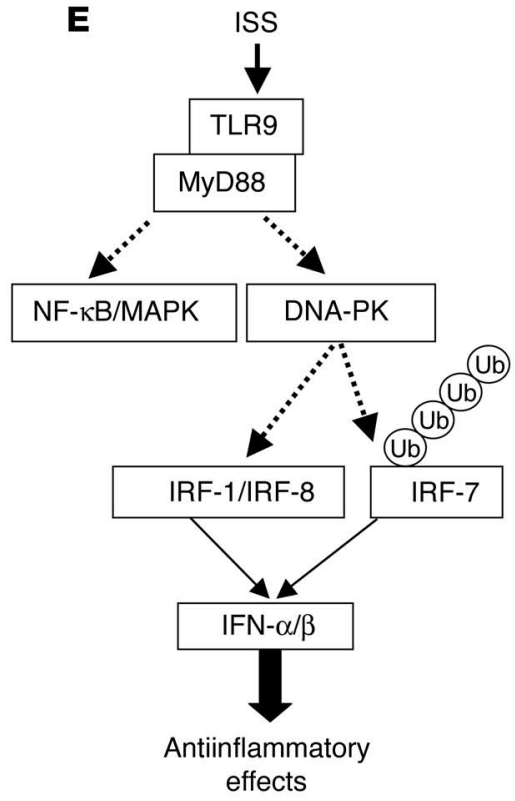

Figure 4

TLR9-activated DNA-PK mediates activation of IRFs and type I IFN via MyD88. (A) BM-PDCs were treated with ISS-1 (10 $\mu \mathrm{g} / \mathrm{ml})$ and nuclear extracts were prepared. NF-кB was detected by EMSA; activation of IRFs, pSTAT1, and pERK, by Western blotting. Results represent 1 of 2 experiments. (B) BM-PDCs (106/ml) from RAG1 $1^{-1-}$ and DNA-PK ${ }^{-1-}$ mice (B6) were stimulated with ISS-1 $(10 \mu \mathrm{g} / \mathrm{ml})$. Cytokine levels in the supernatants were measured 24 hours later. (C) BM-MDCs (106/ml) from $R A G 1^{-/-}$and DNA-PK $K^{-1-}$ mice (B6) were stimulated with ISS-1 (10 $\left.\mu \mathrm{g} / \mathrm{ml}\right)$. Cytokine levels in the supernatants were measured 24 hours later. (D) BM-MDCs $\left(10^{6} / \mathrm{ml}\right)$ from WT and MyD88 $8^{-/-}$mice were stimulated with $10 \mu \mathrm{g} / \mathrm{ml}$ ISS-1 (ISS) or $50 \mathrm{ng} / \mathrm{ml}$ LPS. Activation of DNA-PK was measured by an in vitro kinase assay using GST-p53 as a substrate, as described in Methods. Lysates from BM-MDC from RAG1-/- and SCID mice were stimulated with ISS-1 (10 $\mu \mathrm{g} / \mathrm{ml})$ and then were subjected to immunoprecipitation with anti-ubiquitin, SDS-PAGE, and immunoblotting with anti-IRF-7. Ub-, ubiquitinated. (E) Proposed localization of DNA-PK in the TLR9 signaling pathway. Ub, ubiquitin.

damage and inflammatory cell infiltration were added, resulting in a total scoring range of $0-12$.

\section{Determination of cytokine levels}

ELISA kits were used to determine the levels of IFN- $\gamma$, IL-6, IL-12 p40, IL-10 (BD Biosciences - Pharmingen), and RANTES (R\&D Biosystems). To measure type I IFN levels, we initially compared an ELISA kit (PBL) with a bioassay that is based on an antiviral protection assay (37). As the bioassay was much more sensitive than the currently available mIFN- $\alpha$ ELISA (see Supplemental Figures 1 and 2), we used it in the various studies described here to determine type I IFN levels. Recombinant mIFN- $\beta$ (Chemicon International) was used in the bioassay as a standard.

\section{Signaling assays}

Electrophoretic mobility-shift assay. Nuclear extracts were prepared from BM-MDCs and BM-PDCs that had been stimulated with ISS-ODN or

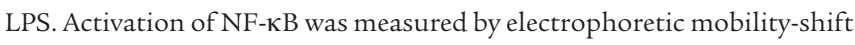
assay as described (38).

Activation of IRF molecules. Levels of different IRFs in the nuclei were measured before and after stimulation with TLR9 and TLR4 agonists using nuclear extracts of BM-MDCs and BM-PDCs. IRFs were detected by Western blotting (39) using antibodies specific for IRF-1, IRF-3, IRF-7, and IRF-8 (Santa Cruz Biotechnology Inc.). IRF-7 ubiquitination status was detected by immunoprecipitation with anti-ubiquitin (Santa Cruz Biotechnology Inc.) followed by immunoblotting with anti-IRF-7 (Santa Cruz Biotechnology Inc.).
Activation of ERK and STAT1. Activation of ERK and STAT1 were assayed with antibodies specific for phosphorylated ERK or STAT1 (40) (Cell Signaling). Levels of $\beta$-actin (Sigma-Aldrich) were used for normalization of protein loading.

DNA-PK activity. The kinase activity of DNA-PK induced by ISS-ODN or LPS was measured by an in vitro kinase assay using GST-p53 as a substrate $(20,41)$. DNA-PK from BM-MDCs and BM-PDCs was immunoprecipitated using anti-DNA-PK (NeoMarkers) before and after ISS-ODN or LPS stimulation, and immune complexes were incubated for 30 minutes with GST-p53 and ${ }^{32} \mathrm{P}-\gamma$-ATP. The reaction samples were then separated by electrophoresis (SDS-PAGE) and were analyzed by autoradiography.

Statistical analysis. Data are expressed as mean \pm SE. Statistical analysis for significant differences was performed according to the Student's $t$ test for unpaired data. A $P$ value less than 0.05 was considered significant.

\section{Acknowledgments}

We thank Lucinda Beck and Jane Uhle for editorial assistance. This work is supported by NIH grants AI40682, DK35108, AR47360, and RR17030.

Received for publication August 10, 2004, and accepted in revised form December 7, 2004.

Address correspondence to: Eyal Raz, Department of Medicine, University of California, San Diego, La Jolla, California 92093-0663, USA. Phone: (858) 534-5444; Fax: (858) 534-5399; E-mail: eraz@ucsd.edu. 
1. Strober, W., Fuss, I.J., and Blumberg, R.S. 2002. The immunology of mucosal models of inflammation. Annu. Rev. Immunol. 20:495-549.

2. Singh, B., et al. 2001. Control of intestinal inflammation by regulatory $\mathrm{T}$ cells. Immunol. Rev. 182:190-200.

3. Podolsky, D.K. 2002. Inflammatory bowel disease. N. Engl. J. Med. 347:417-429.

4. Bouma, G., and Strober, W. 2003. The immunological and genetic basis of inflammatory bowel disease. Nat. Rev. Immunol. 3:521-533.

5. Hemmi, H., et al. 2000. A Toll-like receptor recognizes bacterial DNA. Nature. 408:740-745.

6. Krieg, A.M. 2002. CPG motifs in bacterial DNA and their immune effects. Annu. Rev. Immunol. 20:709-760.

7. Roman, M., et al. 1997. Immunostimulatory DNA sequences function as $\mathrm{T}$ helper-1-promoting adjuvants. Nat. Med. 3:849-854.

8. Horner, A.A., et al. 1998. Immunostimulatory DNA is a potent mucosal adjuvant. Cell. Immunol. 190:77-82.

9. Klinman, D.M. 2004. Immunotherapeutic uses of CpG oligodeoxynucleotides. Nat. Rev. Immunol. 4:249-258.

10. Rachmilewitz, D., et al. 2002. Immunostimulatory DNA ameliorates experimental and spontaneous murine colitis. Gastroenterology. 122:1428-1441

11. Rachmilewitz, D., et al. 2004. Toll-like receptor 9 signaling mediates the anti-inflammatory effects of probiotics in murine experimental colitis. Gas troenterology. 126:520-528.

12. Dieleman, L.A., et al. 1994. Dextran sulfate sodiuminduced colitis occurs in severe combined immunodeficient mice. Gastroenterology. 107:1643-1652.

13. Taniguchi, T., Ogasawara, K., Takaoka, A., and Tanaka, N. 2001. IRF family of transcription factors as regulators of host defense. Annu. Rev Immunol. 19:623-655.

14. Honda, K., et al. 2004. Role of a transductionaltranscriptional processor complex involving MyD88 and IRF-7 in Toll-like receptor signaling. Proc. Natl. Acad. Sci. U. S. A. 101:15416-15421.

15. Kawai, T., et al. 2004. Interferon-alpha induction through Toll-like receptors involves a direct interaction of IRF7 with MyD88 and TRAF6. Nat. Immunol. 5:1061-1068.
16. Beutler, B. 2004. Innate immunity: an overview. Mol. Immunol. 40:845-859.

17. Takeda, K., Kaisho, T., and Akira, S. 2003. Toll-like receptors. Annu. Rev. Immunol. 21:335-376.

18. Kopp, E., and Medzhitov, R. 2003. Recognition of microbial infection by Toll-like receptors. Curr. Opin. Immunol. 15:396-401.

19. Tsujimura, H., et al. 2004. Toll-like receptor 9 signaling activates NF-kappaB through IFN regulatory factor-8/IFN consensus sequence binding protein in dendritic cells. J. Immunol. 172:6820-6827.

20. Tsujimura, H., Tamura, T., and Ozato, K. 2003. Cutting edge: IFN consensus sequence binding protein/IFN regulatory factor 8 drives the development of type I IFN-producing plasmacytoid dendritic cells. J. Immunol. 170:1131-1135.

21. Chu, W., et al. 2000. DNA-PKcs is required for activation of innate immunity by immunostimulatory DNA. Cell. 103:909-918.

22. Karpova, A.Y., Trost, M., Murray, J.M., Cantley, L.C., and Howley, P.M. 2002. Interferon regulatory factor-3 is an in vivo target of DNA-PK. Proc. Natl. Acad. Sci. U. S. A. 99:2818-2823.

23. Bray, M. 2001. The role of the Type I interferon response in the resistance of mice to filovirus infection. J. Gen. Virol. 82:1365-1373.

24. Verthelyi, D., Ishii, K.J., Gursel, M., Takeshita, F., and Klinman, D.M. 2001. Human peripheral blood cells differentially recognize and respond to two distinct CPG motifs. J. Immunol. 166:2372-2377.

25. Hemmi, H., Kaisho, T., Takeda, K., and Akira, S. 2003. The roles of Toll-like receptor 9, MyD88, and DNA-dependent protein kinase catalytic subunit in the effects of two distinct CpG DNAs on dendritic cell subsets. J. Immunol. 170:3059-3064.

26. Biron, C.A. 2001. Interferons alpha and beta as immune regulators--a new look. Immunity. 14:661-664

27. Banchereau, J., Pascual, V., and Palucka, A.K. 2004. Autoimmunity through cytokine-induced dendritic cell activation. Immunity. 20:539-550.

28. Teige, I., et al. 2003. IFN-beta gene deletion leads to augmented and chronic demyelinating experimental autoimmune encephalomyelitis. J. Immunol. 170:4776-4784.

29. Floris, S., et al. 2002. Interferon-beta directly influ- ences monocyte infiltration into the central nervous system. J. Neuroimmunol. 127:69-79.

30. Papadakis, K.A., and Targan, S.R. 2000. Role of cytokines in the pathogenesis of inflammatory bowel disease. Annu. Rev. Med. 51:289-298.

31. Nikolaus, S., et al. 2003. Interferon beta-1a in ulcerative colitis: a placebo controlled, randomised, dose escalating study. Gut. 52:1286-1290.

32. Rakoff-Nahoum, S., Paglino, J., Eslami-Varzaneh, F., Edberg, S., and Medzhitov, R. 2004. Recognition of commensal microflora by toll-like receptors is required for intestinal homeostasis. Cell. 118:229-241.

33. Beamish, H.J., et al. 2000. The C-terminal conserved domain of DNA-PKcs, missing in the SCID mouse, is required for kinase activity. Nucleic Acids Res. 28:1506-1513.

34. Kurimasa, A., et al. 1999. Catalytic subunit of DNAdependent protein kinase: impact on lymphocyte development and tumorigenesis. Proc. Natl. Acad. Sci. U. S. A. 96:1403-1408.

35. Martin-Orozco, E., et al. 1999. Enhancement of antigen-presenting cell surface molecules involved in cognate interactions by immunostimulatory DNA sequences. Int. Immunol. 11:1111-1118.

36. Datta, S.K., et al. 2003. A subset of Toll-like receptor ligands induces cross-presentation by bone marrowderived dendritic cells. J. Immunol. 170:4102-4110.

37. Hoebe, K., et al. 2003. Identification of Lps 2 as a key transducer of MyD88-independent TIR signaling. Nature. 424:743-748.

38. Lee, J., Mira-Arbibe, L., and Ulevitch, R.J. 2000. TAK1 regulates multiple protein kinase cascades activated by bacterial lipopolysaccharide. J. Lenkoc. Biol. 68:909-915.

39. Au, W.C., and Pitha, P.M. 2001. Recruitment of multiple interferon regulatory factors and histone acetyltransferase to the transcriptionally active interferon a promoters. J. Biol. Chem. 276:41629-41637.

40. Hsu, L.C., et al. 2004. The protein kinase PKR is required for macrophage apoptosis after activation of Toll-like receptor 4. Nature. 428:341-345.

41. Woo, R.A., McLure, K.G., Lees-Miller, S.P., Rancourt, D.E., and Lee, P.W. 1998. DNA-dependent protein kinase acts upstream of p53 in response to DNA damage. Nature. 394:700-704 